\title{
Effect of dual growth factor delivery on chondrogenic differentiation of rabbit marrow mesenchymal stem cells encapsulated in injectable hydrogel composites
}

\author{
Hansoo Park, ${ }^{1}$ Johnna S. Temenoff, ${ }^{2}$ Yasuhiko Tabata, ${ }^{3}$ Arnold I. Caplan, ${ }^{4}$ Robert M. Raphael, ${ }^{1}$ \\ John A. Jansen, ${ }^{5}$ Antonios G. Mikos ${ }^{1}$ \\ ${ }_{1}^{1}$ Department of Bioengineering, Rice University, MS-142, P.O. Box 1892, Houston, Texas 77251-1892 \\ ${ }^{2}$ Wallace H. Coulter Department of Biomedical Engineering, Georgia Institute of Technology, 313 Ferst Dr., \\ Atlanta, Georgia 30332-0535 \\ ${ }^{3}$ Department of Biomaterials, Field of Tissue Engineering, Institute for Frontier Medical Sciences, Kyoto University, \\ 53 Kawara-cho Shogoin, Sakyo-ku, Kyoto 606-8507, Japan \\ ${ }^{4}$ Skeletal Research Center, Department of Biology, Case Western Reserve University, Cleveland, Ohio 44106-7080 \\ ${ }^{5}$ Department of Periodontology and Biomaterials, Radboud University Nijmegen Medical Center, \\ 309 P.O. Box 9101, 6500 HB Nijmegen, The Netherlands
}

Received 26 June 2007; revised 21 December 2007; accepted 4 January 2008

Published online 31 March 2008 in Wiley InterScience (www.interscience.wiley.com). DOI: 10.1002/jbm.a.31948

\begin{abstract}
An injectable hydrogel composite consisting of oligo(poly(ethylene glycol)fumarate) (OPF) and gelatin microparticles has been developed as a novel carrier system for cells and growth factors. Rabbit marrow mesenchymal stem cells (MSCs) and gelatin microparticles (MPs) loaded with insulin-like growth factor-1 (IGF-1), transforming growth factor- $\beta 1$ (TGF- $\beta 1$ ), or a combination of both growth factors were mixed with OPF, a poly(ethylene glycol)-diacrylate crosslinker and the radical initiators ammonium persulfate and $N, N, N^{\prime}, N^{\prime}$-tetramethylethylenediamine, and then crosslinked at $37^{\circ} \mathrm{C}$ for $8 \mathrm{~min}$ to form hydrogel composites. Hydrogel composites encapsulating rabbit marrow MSCs and blank MPs served as controls. At day 14, confocal fluorescent images of OPF hydrogels showed a strong aggregation of rabbit marrow MSCs when encapsulated with IGF-1-loaded MPs with or without TGF- $\beta 1$-loaded MPs. Quantitative RT-PCR results showed that rabbit marrow MSCs encapsulated with MPs
\end{abstract}

loaded with TGF- $\beta 1$ or both TGF- $\beta 1$ and IGF- 1 had a significant increase in the expression of chondrocyte-specific genes such as collagen type II and aggrecan at day 14 as compared with the control group. Specifically, samples with both TGF- 31 -loaded MPs and IGF-1-loaded MPs exhibited a $121 \pm 20$-fold increase of type II collagen gene expression and a $71 \pm 24$-fold increase of aggrecan gene expression after 14 days of in vitro culture as compared with controls at day 0 . These results suggest that hydrogel composites based on OPF and gelatin microparticles have great potential as carriers for MSCs and multiple growth factors for cartilage tissue engineering applications. (C) 2008 Wiley Periodicals, Inc. J Biomed Mater Res 88A: 889-897, 2009

Key words: cartilage tissue engineering; marrow mesenchymal stem cells; gelatin microparticles; injectable hydrogels; TGF- $\beta 1$; IGF-1

\section{INTRODUCTION}

Articular cartilage tissue is an avascular tissue withstanding both compressive loads and friction in the joint. ${ }^{1}$ The complicated structure of articular cartilage often leads to failure in self repair and necessitates the need for intervention for the treatment of cartilage defects. The use of mesenchymal stem cells

Correspondence to: A. G. Mikos; e-mail: mikos@rice.edu Contract grant sponsor: National Institutes of Health; contract grant number: R01 AR48756

(C) 2008 Wiley Periodicals, Inc.
(MSCs) has been proposed as a promising cell therapy to restore cartilage defects, since MSCs can be expanded multiple times without losing their capacity to differentiate into chondrocytes. ${ }^{2}$ However, the delivery of MSCs to cartilage defects still remains a challenge.

To date, various types of scaffolds made of synthetic or natural polymers have been examined to establish a favorable environment for chondrogenic differentiation of MSCs. ${ }^{3-5}$ Hydrogels are especially attractive for the delivery of MSCs as they can retain a high amount of water, and thus facilitate the transport of nutrients to cells encapsulated in hydrogel scaffolds. ${ }^{1,6}$ In addition, the administration and 
release of bioactive factors required for differentiation of MSCs are easily modulated by altering the macromolecular structure of hydrogels. ${ }^{7}$ A novel injectable and biodegradable oligo(poly(ethylene glycol)fumarate) (OPF) hydrogel has been studied for the delivery of cells and growth factors for cartilage tissue engineering applications.

Transforming growth factors- $\beta 1$ (TGF- $\beta 1$ ), known to induce chondrogenic differentiation of MSCs, was successfully incorporated into OPF hydrogel composites including gelatin microparticles (MPs) and released in a controlled manner depending on the crosslinking characteristics of the hydrogel composites. ${ }^{8}$ An in vitro study with OPF hydrogels encapsulating chondrocytes and TGF- $\beta 1$-loaded MPs showed that TGF- $\beta 1$ released from the MPs promoted cell proliferation while cells maintained the chondrocytic phenotype. ${ }^{9}$ In addition, when rabbit marrow MSCs were encapsulated in hydrogels with TGF- $\beta 1$-loaded MPs, an upregulation of chondrocyte-specific genes such as collagen type II and aggrecan was observed. ${ }^{10}$

OPF hydrogel composites were also used for the delivery of IGF-1, known to increase colony formation and extracellular matrix production of chondrocytes $^{7,11}$ along with TGF- $\beta 1 .{ }^{12}$ Moreover, bilayered OPF hydrogels encapsulating gelatin microparticles loaded with TGF- $\beta 1$ and/or IGF- 1 in each layer were implanted into cartilage defects of rabbits and demonstrated the potential of OPF hydrogel composites as delivery vehicles for multiple growth factors. ${ }^{13}$

In this study, we investigated the encapsulation of rabbit marrow MSCs in OPF hydrogel composites containing gelatin microparticles loaded with TGF$\beta 1$, IGF-1, or both TGF- $\beta 1$ and IGF-1, and examined the effect of dual growth factor delivery on the chondrogenic differentiation of encapsulated cells with confocal microscopy, biochemical assays, and realtime PCR.

\section{MATERIALS AND METHODS}

\section{OPF synthesis and characterization}

OPF was synthesized from fumaryl chloride and PEG of nominal molecular weight 10,000 according to a previously established method. ${ }^{14}$ Oligomer molecular weight was determined by gel permeation chromatography (GPC; Model 410; Waters, Milford, PA) using a refractive index detector $(n=3)$. Purified OPF was stored at $-20^{\circ} \mathrm{C}$ and sterilized prior to use by exposure to ethylene oxide for $14 \mathrm{~h}$.

\section{Gelatin microparticle preparation}

Gelatin microparticles were fabricated from acidic gelatin (Nitta Gelatin, Osaka, Japan) following established procedures. $^{9}$ Briefly, a gelatin solution was prepared by dissolving $5 \mathrm{~g}$ gelatin in $45 \mathrm{~mL}$ distilled and deionized water $\left(\mathrm{ddH}_{2} \mathrm{O}\right)$ at $60^{\circ} \mathrm{C}$. Then, this solution was added dropwise to $250 \mathrm{~mL}$ chilled olive oil while stirring at 500 rpm. After $30 \mathrm{~min}, 100 \mathrm{~mL}$ chilled acetone $\left(4^{\circ} \mathrm{C}\right)$ was added to the emulsion. After an additional $60 \mathrm{~min}$, the microparticles were collected by filtration and washed with acetone. These microparticles were then crosslinked in a $0.1 \mathrm{wt} \%$ Tween 80 (Sigma, St. Louis, MO) solution with $10 \mathrm{mM}$ glutaraldehyde (GA) (Sigma) while stirring at $500 \mathrm{rpm}$ at $15^{\circ} \mathrm{C}$. After $15 \mathrm{~h}$, crosslinked microparticles were collected by filtration, washed with $\mathrm{ddH}_{2} \mathrm{O}$, and then agitated in $25 \mathrm{mM}$ glycine solution for $1 \mathrm{~h}$ to inactivate any unreacted GA. These microparticles were collected by filtration, washed with $\mathrm{ddH}_{2} \mathrm{O}$, and then lyophilized overnight. Finally, dried microparticles were sieved to obtain particles of 50-100 $\mu \mathrm{m}$ diameter and sterilized with ethylene oxide for $14 \mathrm{~h}$.

\section{Rabbit marrow MSC isolation and preculture}

Rabbit marrow MSCs were isolated from the femur of 4week-old rabbits as previously described. ${ }^{2,10}$ Briefly, after anesthesia, rabbit bone marrow was collected into a $10-\mathrm{mL}$ syringe containing $5000 \mathrm{U}$ of heparin. The bone marrow was then filtered through a cell strainer $(40 \mu \mathrm{m})$ and cultured in DMEM-LG supplemented medium containing $10 \% \mathrm{v} / \mathrm{v}$ fetal bovine serum (Gemini, Calabasas, CA), $250 \mu \mathrm{g} / \mathrm{L}$ fungizone, $100 \mathrm{mg} / \mathrm{L}$ ampicillin, and $50 \mathrm{mg} / \mathrm{L}$ gentamicin (Invitrogen) for 2 weeks. In an effort to reduce any interanimal variation, a pool of rabbit marrow MSCs from a total of 6 rabbits were collected together, placed in medium containing 20\% FBS and 10\% dimethyl sulfoxide (DMSO), and cryopreserved in liquid nitrogen prior to use. For experiments, cryopreserved cells were thawed at $37^{\circ} \mathrm{C}$, seeded in T-75 flasks, and expanded for 14 days of culture in DMEM supplemented medium containing $10 \%$ $\mathrm{v} / \mathrm{v}$ fetal bovine serum (Gemini), $250 \mu \mathrm{g} / \mathrm{L}$ fungizone, $100 \mathrm{mg} / \mathrm{L}$ ampicillin, and $50 \mathrm{mg} / \mathrm{L}$ gentamicin. Cells from one preparation were used in all experiments and were cultured up to passage three before the encapsulation process.

\section{Encapsulation of rabbit marrow MSCs and gelatin microparticles}

Before encapsulation, gelatin microparticles were sterilized with ethylene oxide for $14 \mathrm{~h}$. Then, sterilized MPs were loaded with either IGF-1 or TGF- $\beta 1$ by immersing them in aqueous solution containing either IGF-1 or TGF$\beta 1$ at $\mathrm{pH} 7.4$ and incubating them at $4{ }^{\circ} \mathrm{C}$ for $15 \mathrm{~h}$ according to established methods. ${ }^{12}$ To achieve the final concentration of $10 \mathrm{ng}$ TGF- $\beta 1 / \mathrm{mL}$ and $100 \mathrm{ng}$ IGF- $1 / \mathrm{mL}$ based on the medium, $55 \mu \mathrm{L}$ of aqueous solution with either $7.2 \mathrm{ng}$ TGF- $\beta 1 / \mu \mathrm{L}$ or $72 \mathrm{ng}$ IGF- $1 / \mu \mathrm{L}$ were loaded into $11 \mathrm{mg}$ of gelatin microparticles. Eleven milligrams of blank MPs were also prepared.

Following this incubation period, isolated rabbit marrow MSCs and gelatin microparticles were encapsulated in crosslinked OPF. In a typical experiment, $0.1 \mathrm{~g}$ of OPF and 
$0.05 \mathrm{~g}$ of the crosslinking agent poly(ethylene glycol) diacrylate (PEG-DA; nominal MW 3400, Nektar Therapeutics, Huntsville, AL) were combined in $300 \mu \mathrm{L}$ PBS and mixed with $22 \mathrm{mg}$ of microparticles partially swollen in $55 \mu \mathrm{L}$ of PBS containing $7.2 \mathrm{ng}$ TGF- $\beta 1 / \mu \mathrm{L}$ and $55 \mu \mathrm{L}$ of PBS containing $72 \mathrm{ng}$ IGF-1/ $\mathrm{LL}$. Equal volumes $(46.8 \mu \mathrm{L})$ of the thermal radical initiators, $25 \mathrm{mM}$ ammonium persulfate (APS) and $25 \mathrm{mM} \mathrm{N}, N, N^{\prime}, N^{\prime}$-tetramethylethylenediamine (TEMED) in PBS were then added. After this mixture was vortexed, a $168-\mu \mathrm{L}$ PBS suspension containing 6.7 million cells was added to achieve a cell concentration of 10 million cells $/ \mathrm{mL}$ in the final suspension. After gentle mixing, the suspension was quickly injected into Teflon molds (6 $\mathrm{mm}$ diameter, $1 \mathrm{~mm}$ thickness) followed by incubation at $37^{\circ} \mathrm{C}$ for $8 \mathrm{~min}$. Final gel constructs were transferred into a 12 -well tissue culture plates containing $2.5 \mathrm{~mL}$ medium in each well. The medium was changed every 3 days. At days 3, 7, and 14, samples were collected for biochemical assays $(n=4)$, quantitative reverse transcriptasepolymerase chain reaction (RT-PCR) $(n=4)$, and histological analysis $(n=2)$. Samples at day 0 were collected immediately after the encapsulation process and used as a control for RT-PCR. To compare the effect of dual growth factor delivery to that of single growth factor delivery on encapsulated rabbit marrow MSCs, hydrogel composites encapsulating either IGF-1-loaded MPs or TGF- $\beta 1$-loaded MPs were also prepared. Additionally, hydrogel composites with blank MPs were also prepared at each time point.

\section{Confocal fluorescence microscopy}

Confocal fluorescence microscopy (Zeiss LSM 510, Thornwood, NY) was used to visualize rabbit marrow MSCs encapsulated in OPF hydrogel composites at day 14. Specifically, rabbit marrow MSCs complexed with a Live/ Dead reagent (combination of $4 \mu M$ ethidium homodimer1 (EthD-1) and $2 \mu M$ calcein acetoxymethyl ester (calcein $\mathrm{AM}$ ) were excited using a $488 \mathrm{~nm}$ laser, and images were collected using emission from a 500-550 nm bandpass filter for Live staining and a $650 \mathrm{~nm}$ long pass filter for Dead staining with a resolution of $10 \times$. To visualize cell morphology in hydrogels, samples were stained with rhodamine phalloidin for $1 \mathrm{~h}$ in the incubator after being rinsed with PBS and incubated with $0.5 \%$ Triton for $30 \mathrm{~min}$. Then, samples were excited using a $540 \mathrm{~nm}$ laser and images were collected using emission from a $565 \mathrm{~nm}$ long pass filter with a resolution of $20 \times$. Three dimensional images of rabbit marrow MSCs were also collected using a series of $Z$ optical sections. The thickness between each section was $25 \mu \mathrm{m}$ and the projection images were acquired by overlapping each slice in the $z$ sections using Zeiss Aim software. Single hydrogel constructs were analyzed for each group examined.

\section{DNA assay}

At each time point, samples and cell-free hydrogels were removed from medium, rinsed in $2 \mathrm{~mL}$ PBS, homogenized with a pellet grinder (Fisher Scientific), and digested in $500 \mu \mathrm{L}$ of a proteinase $\mathrm{K}$ solution $(1 \mathrm{mg} / \mathrm{mL}$ proteinase K (Sigma-Aldrich), $10 \mu \mathrm{g} / \mathrm{mL}$ pepstatin A (Sigma-Aldrich), and $185 \mu \mathrm{g} / \mathrm{mL}$ iodoacetamide (SigmaAldrich) in tris-EDTA solution $(6.055 \mathrm{mg} / \mathrm{mL}$ Tris(hydroxymethyl aminomethane) (Sigma-Aldrich), $0.372 \mathrm{mg} / \mathrm{mL}$ EDTA (Sigma-Aldrich), pH 7.6 adjusted by $\mathrm{HCl}$ ) at $60^{\circ} \mathrm{C}$ for $16 \mathrm{~h}$. After collection and digestion of all samples and cell-free hydrogels, specimens underwent three repetitions of a freeze/thaw/sonication cycle $\left(30 \mathrm{~min}\right.$ at $-80^{\circ} \mathrm{C}$, $30 \mathrm{~min}$ at room temperature, $30 \mathrm{~min}$ of sonication) for complete extraction of DNA from the cell cytoplasm. DNA assays were then run for each experimental and control group at each time point. DNA content was calculated by measuring double-stranded DNA content using the PicoGreen assay (Molecular Probes, Eugene, OR) according to the manufacturer's instructions. The fluorescence of cellfree hydrogels was subtracted from the fluorescence values of experimental groups to account for fluorescence of the material alone.

\section{Quantitative RT-PCR for gene expression}

Total RNA was extracted from hydrogel composites at each time point via the RNeasy Mini Kit (Qiagen, Hilden, Germany). Briefly, hydrogels were transferred into RNA lysis buffer solution and homogenized by gentle pipetting. The homogenized solution was purified using a Qiagen shredder column and total RNA was extracted with the RNeasy Mini Kit. RNA samples were then reverse-transcribed to cDNA using Oligo dT primers and superscript III transcriptase. The final cDNA was then subjected to real-time PCR (ABI Biomed 7300 Real-Time PCR System) to determine the expression of genes for type II collagen genes, aggrecan, and type I collagen. The results from realtime quantitative PCR were analyzed by relative quantification. ${ }^{15}$ Relative quantification compares the PCR signals of a gene of interest in a treatment group to that of an untreated control (the control can be the value at time zero for a time-course study) after the gene of interest is normalized to a housekeeping gene. For these studies, glyceraldehyde-3-phosphatase dehydrogenase (GAPDH) was used as the housekeeping gene, and OPF hydrogel composites with blank MPs encapsulating rabbit marrow MSCs immediately after encapsulation (day 0) were used as the control group. The sequence of primers for GAPDH, type I collagen, type II collagen, and aggrecan were as follows: GAPDH: 5-TCACCATCTTCCAGGAGCGA-3, 5-CACAAT GCCGAAGTGGTCGT-3; type I collagen gene: 5-CTTCTG GCCCTGCTGGAAAGGATG-3, 5-CCCGGATACAGGTTTC GCCAGTAG-3; type II collagen gene: 5'-AACACTGCCA ACGTCCAGAT-3', 5'-CTGCAGCACGGTATAGGTGA-3'; Aggrecan: 5'-GCTACGGAGACAAGGATGAGTTC-3', 5' CGTAAAAGACCTCACCCTCCAT-3'.

\section{GAG assay}

Glycosaminoglycan (GAG) content was determined using a biochemical assay, the dimethylmethylene blue dye (DMMB) assay (Sigma-Aldrich), as previously described. ${ }^{16}$ Samples prepared for DNA assay were used 
for GAG assay, and GAG/DNA content was measured for each sample. Upon DMMB binding to GAG, a pink color is produced that allows for quantification of GAG by measuring absorbance at $520 \mathrm{~nm}$. GAG content in hydrogels was calculated by comparison to a curve generated from standards of known amounts of chondroitin sulfate (Sigma-Aldrich). A microplate reader (BIO-TEK Instrument, Winooski, VT) was utilized for both the absorbance and fluorescence measurements.

\section{Histology}

Histology samples were prepared as previously described.9 Briefly, samples from each time point were rinsed in $2 \mathrm{~mL}$ PBS for $1 \mathrm{~h}$ and then fixed in 10\% neutral buffered formalin (Sigma-Aldrich). After fixation, these samples were dehydrated by immersion in a series of ethanol solutions (70, 80, 85, 90, 95, and 100\%) and xylene solutions in ethanol (50 and 100\%). Specimens were then embedded in paraffin and cross-sectioned to a thickness of $20 \mu \mathrm{m}$ using a microtome (Microm, Walldorf, Germany). Sections from all groups were simultaneously stained with Safranin-O. Images were acquired with a light microscope (Eclipse E600; Nikon, Melvile, NY) equipped with a video camera (3CCD Color Video Camera DXC-950P; Sony, Park Ridge, NJ).

\section{Statistical analysis}

DNA, GAG, and gene expression are reported as means \pm SD. Repetitive ANOVA and Tukey's multiple comparison tests were used to determine possible significant differences $(p<0.05)$ in the DNA and GAG contents for each group.

\section{RESULTS}

OPF hydrogel composites were previously examined as an injectable carrier for delivery of both TGF- $\beta 1$ and IGF-1 to cartilage defects. ${ }^{12,17}$ In vitro release kinetics data showed that both growth factors exhibited a similar burst release in PBS when they were incorporated into acidic gelatin microparticles. ${ }^{12}$ Acidic gelatin microparticles loaded with IGF-1 had an approximate cumulative burst release of $22 \%$ after 3 days and an approximate cumulative release of $32 \%$ after 28 days, while the corresponding cumulative releases for TGF- $\beta 1$ were $26 \%$ after 3 days and $45 \%$ after 28 days. ${ }^{12}$ When microparticles loaded with growth factors were encapsulated within OPF hydrogels, an increased retention of the growth factors was observed as compared with both nonencapsulated loaded microparticles and OPF hydrogels loaded with growth factors in the absence of microparticles. ${ }^{12}$ Accordingly, this study was designed to assess the effects of dual growth factor delivery on rabbit MSCs encapsulated in hydrogel composites including microparticles loaded with TGF- $\beta 1$, IGF-1, or their combination.

\section{Confocal fluorescence microscopy}

At 14 days, the viability and distribution of encapsulated rabbit marrow MSCs were qualitatively examined by confocal fluorescence microscopy. Cells were stained with Live/Dead assay kit containing calcein AM and ethidium homodimer-1 for cell viability and distribution [Fig. 1(A-C)]. Calcein AM stains live cells with green fluorescence, and ethidium homodimer-1 stains dead cells with red fluorescence. Cells were also stained with rhodamine phalloidin to visualize cell morphology in hydrogel composites [Fig. 1(D-F)]. As shown in Figure 1(AC), when rabbit marrow MSCs (green color) were encapsulated in OPF hydrogel composites, they were well distributed throughout the hydrogel. An apparent increase in the aggregation of rabbit marrow MSCs was noted in groups containing IGF-1-loaded MPs [Fig. 1(B,C)]. Figure 1(D-F) also shows that most of the rabbit marrow MSCs maintained their round morphology. The aggregation of rabbit marrow MSCs is also shown in a Z-axial projection image for a hydrogel composite containing both TGF- $\beta 1$-loaded MPs and IGF-1-loaded MPs after staining with rhodamine phalloidin (Fig. 2).

\section{DNA assay}

DNA content at each time point is depicted in Figure 3. All the groups exhibited a similar decrease in DNA content after 14 days. Groups containing TGF$\beta 1$-loaded MPs had a significant decrease in DNA content starting at day 7 and a significant difference when compared with other groups at day 14 .

\section{Quantitative gene expression analysis}

Quantitative gene expressions for collagen type II, aggrecan, and collagen type I were measured by real-time RT-PCR to assess the expression of chondrocyte-specific genes of encapsulated rabbit marrow MSCs. As shown in Figure 4(A), gene expression for collagen type II followed an increasing trend at day 14 for all groups as compared with the control group (no growth factor at day 0). However, the highest increase in collagen type II gene expression was found in cells encapsulated in hydrogel composites containing TGF- $\beta 1$-loaded MPs and both TGF- $\beta 1$ loaded MPs and IGF-1-loaded MPs. At day 14, hydrogel composites containing TGF- $\beta 1$-loaded MPs and both TGF- $\beta 1$-loaded MPs and IGF-1-loaded MPs 

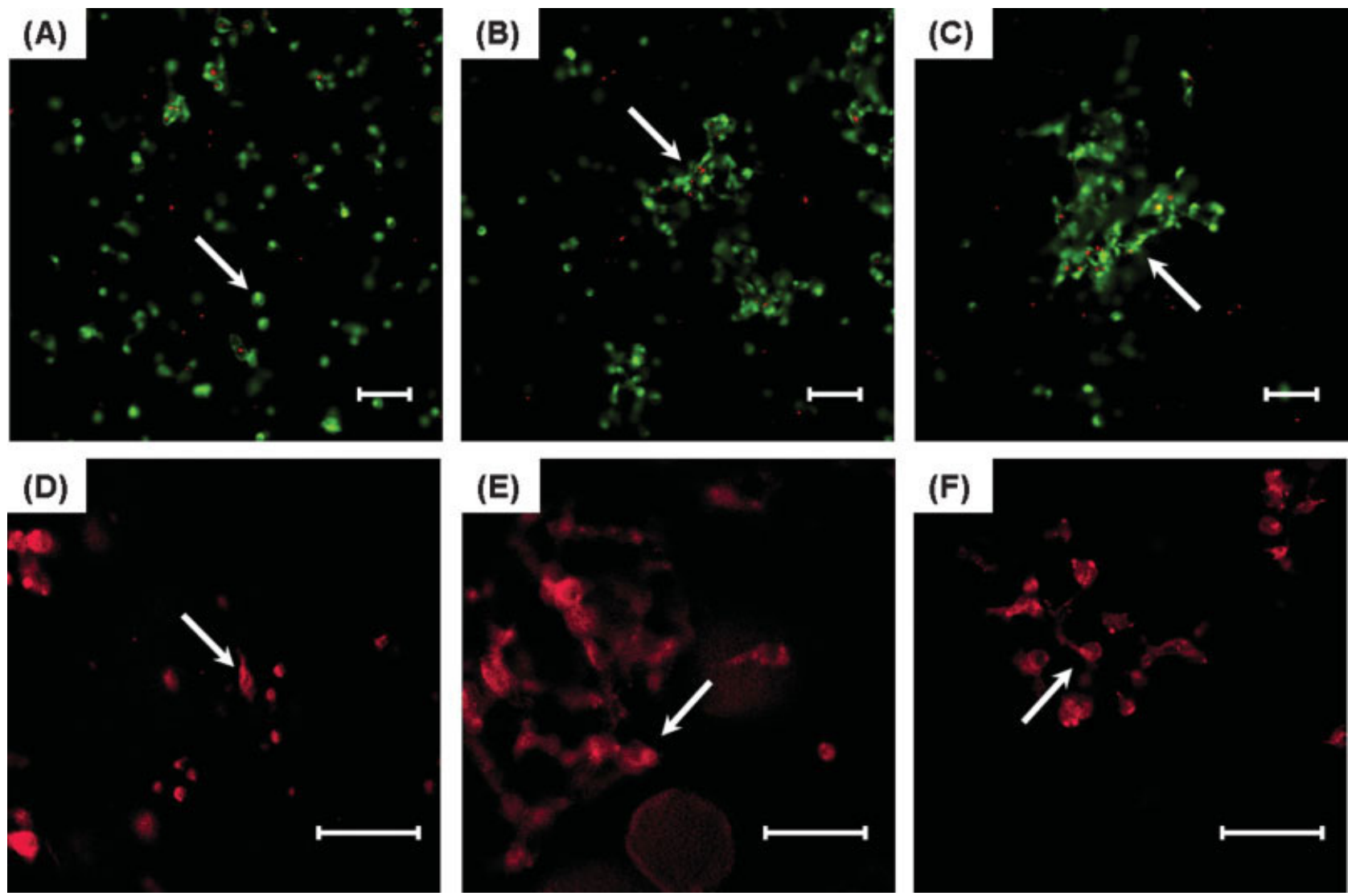

Figure 1. Confocal fluorescence microscopy images of rabbit marrow MSCs encapsulated in OPF hydrogel composites at day 14. Images show rabbit marrow MSCs stained with Live/Dead assay reagents (calcein AM and EthD-1) in (A) OPF hydrogel composites with blank MPs, (B) OPF hydrogel composites with IGF-1-loaded MPs, and (C) OPF hydrogel composites with both IGF-1-loaded MPs and TGF- $\beta 1$-loaded MPs. Additional images are for rabbit marrow MSCs stained with rhodamine phalloidin in (D) OPF hydrogel composites with blank MPs, (E) OPF hydrogel composites with IGF-1loaded MPs, and (F) OPF hydrogel composites with both IGF-1-loaded MPs and TGF- $\beta 1$-loaded MPs. Scale bar represents $100 \mu \mathrm{m}$. Arrows indicate rabbit marrow MSCs in OPF hydrogel composites. [Color figure can be viewed in the online issue, which is available at www.interscience.wiley.com.]
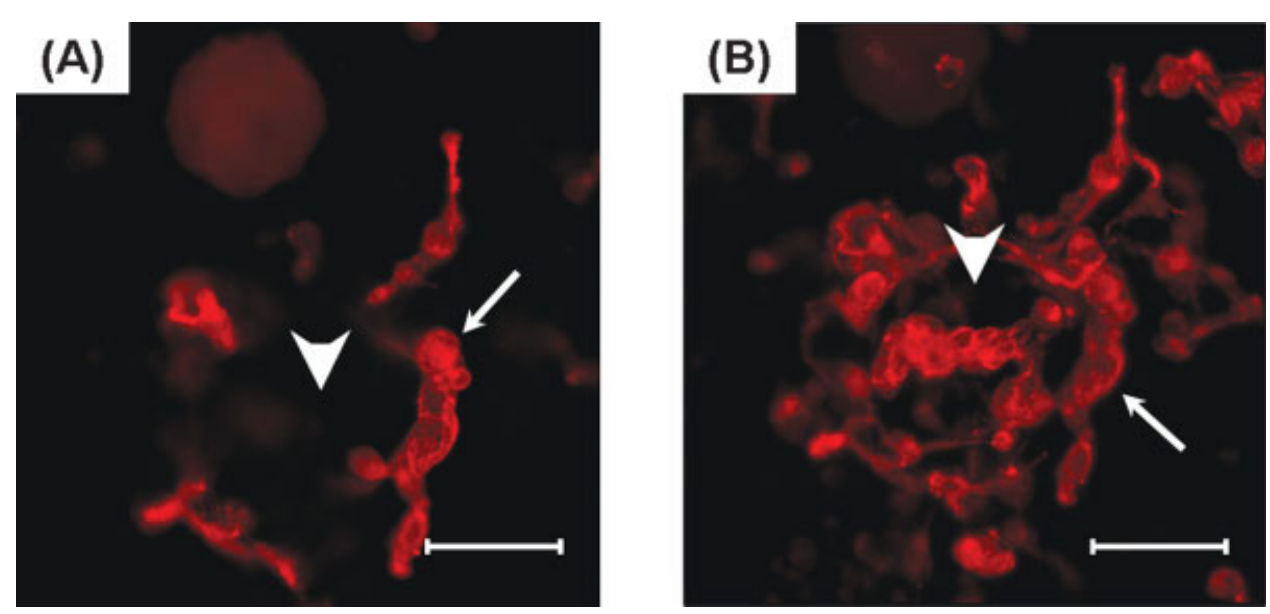

Figure 2. 2D image (A) and z-axial projection stack image (B) of rabbit marrow MSCs in OPF hydrogel composites with both IGF-1-loaded MPs and TGF- 31 -loaded MPs at day 14. Samples were incubated with rhodamine phalloidin solution for $1 \mathrm{~h}$ and imaged with confocal fluorescence microscopy. Scale bar represents $100 \mu \mathrm{m}$. Small arrows indicate rabbit marrow MSCs and large arrows indicate MPs in hydrogel composites. [Color figure can be viewed in the online issue, which is available at www.interscience.wiley.com.] 


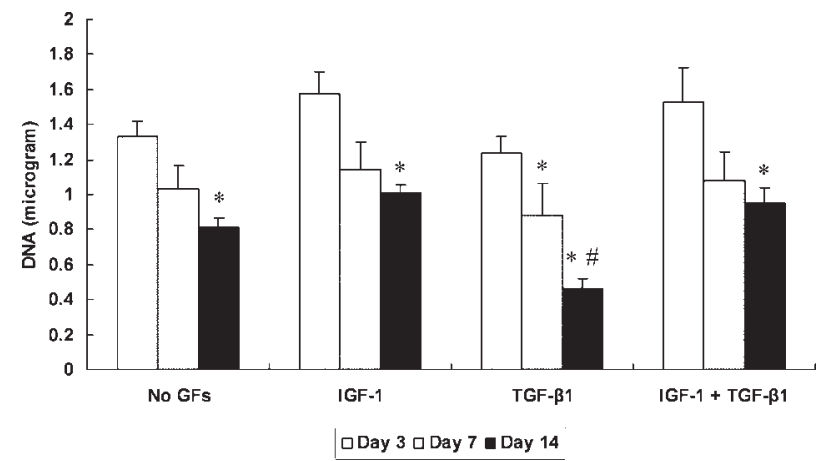

Figure 3. DNA content of OPF hydrogel composites encapsulating rabbit marrow MSCs/blank MPs (No GFs), rabbit marrow MSCs/IGF-1-loaded MPs (IGF-1), rabbit marrow MSCs/TGF- $\beta 1$-loaded MPs (TGF- $\beta 1$ ), or rabbit marrow MSCs/both IGF-1-loaded MPs and TGF- $\beta 1$-loaded MPs (IGF-1 + TGF- $\beta 1$ ) over a 14-day culture period. Samples marked by $\left(^{*}\right)$ exhibited significantly lower DNA content as compared with the same sample type at day $3(p<$ $0.05)$. The symbol (\#) indicates a significant difference in DNA content as compared with the other groups at the same time point $(p<0.05)$. Error bars represent means \pm SD with $n=4$.

exhibited $121 \pm 37$-fold increase and $122 \pm 20$-fold increase in collagen type II gene expression, respectively, when compared with controls.

A significant increase in aggrecan gene expression was observed in groups with TGF- $\beta 1$-loaded MPs. Aggrecan expression showed $75 \pm 66$-fold increase in groups containing TGF- $\beta 1$-loaded MPs and $71 \pm$ 24-fold increase in groups containing both TGF- $\beta 1$ loaded MPs and IGF-1-loaded MPs after 14 days when compared with controls [Fig. 4(B)]. The expression of collagen type I was not significantly different across the culture period except for groups containing IGF-1-loaded MPs only [Fig. 4(C)]. However, in all cases, the fold increases of expression for collagen type I was negligible when compared with the corresponding values for collagen type II and aggrecan.

\section{GAG assay and histology}

As shown in Figure 5, GAG results showed that no significant differences in GAG/DNA content were found between all groups during the 14-day culture period.

The presence of GAG within cell constructs was visualized by Safranin-O staining. Figure 6 displays cross sections of these constructs at days 0 and 14 . Rabbit marrow MSCs (noted by small arrows) and gelatin microparticles (noted by large arrows) were clearly stained with a deep red. Because of the different swelling properties of the OPF hydrogel and the gelatin microparticles, the preparation of samples for histology resulted in tearing of some samples.
Similar to confocal fluorescence microscopy results, cells and MPs were homogenously distributed throughout hydrogel composites, and larger cell aggregates were observed in sections of OPF hydrogel composites containing IGF-1-loaded MPs than in sections of OPF hydrogel composites devoid of IGF1-loaded MPs. However, no apparent differences in GAG staining were found between groups at day 14 .

(A)

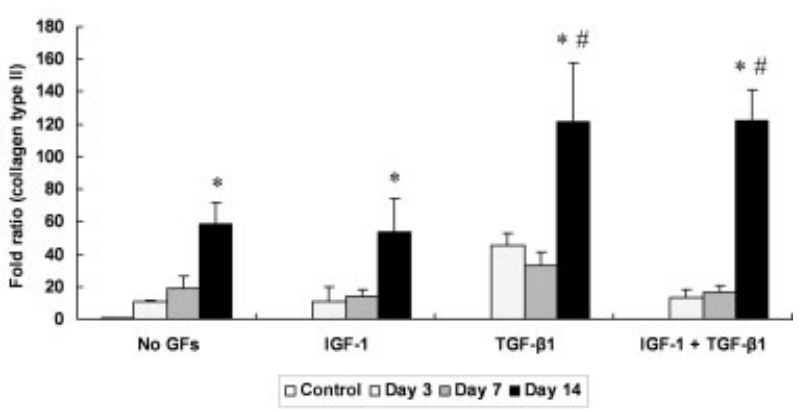

B)

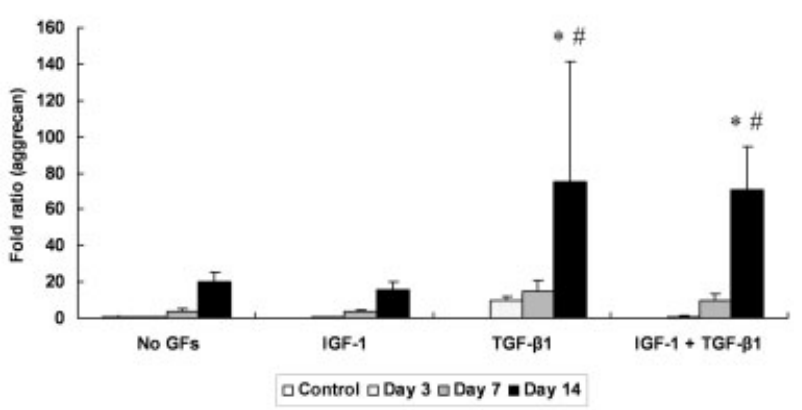

(C)

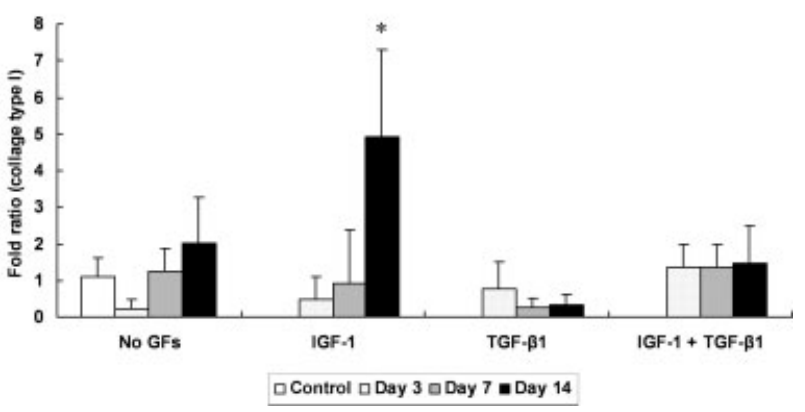

Figure 4. Quantitative analysis of (A) collagen type II, (B) aggrecan, (C) collagen type I gene expression by rabbit marrow MSCs encapsulated in OPF hydrogel composites with blank MPs (No GFs), IGF-1-loaded MPs (IGF-1), TGF$\beta 1$-loaded MPs (TGF- $\beta 1$ ), or both IGF-1-loaded MPs and TGF- $\beta 1$-loaded MPs (IGF-1 + TGF- $\beta 1$ ) over a 14-day culture period. Data are presented as a fold ratio after normalization to GAPDH values. The expression level of controls (day 0 ) is represented as 1 . Within a given hydrogel formulation, significantly higher $(p<0.05)$ gene expression than the day 0 value (control) is noted with $\left(^{*}\right)$. Samples indicated with (\#) had significantly higher gene expression than the other two groups at the same time point $(p<$ 0.05). Error bars represent means \pm SD for $n=4$. 


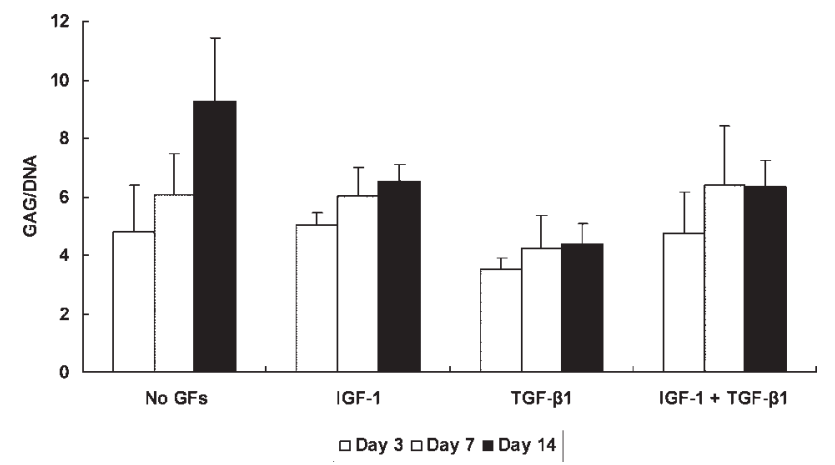

Figure 5. GAG/DNA content of OPF hydrogel composites encapsulating rabbit marrow MSCs/blank MPs (No GFs), rabbit marrow MSCs/IGF-1-loaded MPs (IGF-1), rabbit marrow MSCs/TGF- $\beta 1$-loaded MPs (TGF- $\beta 1$ ), or rabbit marrow MSCs/both IGF-1-loaded MPs and TGF- $\beta 1$-loaded MPs (IGF-1 + TGF- $\beta 1$ ) over a 14-day culture period. No significant differences in GAG/DNA content were found between or within groups over the culture period. Error bars represent means \pm SD for $n=4$.

\section{DISCUSSION}

Several studies have reported that the combination of IGF- 1 and TGF- $\beta 1$ promotes in vitro chondrogenic differentiation of MSCs while maintaining their pro- liferation and GAG production. ${ }^{18,19}$ However, surprisingly, the dual delivery of IGF-1 and TGF- $\beta 1$ via OPF hydrogel composites did not improve cartilage formation when they were used for the repair of osteochondral defects in a rabbit model. ${ }^{13}$ Based on this observation, this study was designed to assess the combined effect of these growth factors (TGF- $\beta 1$ and IGF-1) loaded into gelatin microparticles on the chondrogenic differentiation of rabbit marrow MSCs embedded in OPF hydrogels in vitro. For this study, four different groups were tested as follows: rabbit marrow MSCs and blank MPs encapsulated in OPF, rabbit marrow MSCs and IGF-1-loaded MPs encapsulated in OPF, rabbit marrow MSCs and TGF- $\beta 1-$ loaded MPs encapsulated in OPF, and rabbit marrow MSCs and both IGF-1-loaded MPs and TGF- $\beta 1-$ loaded MPs encapsulated in OPF. The cell seeding density was 10 million cells/mL expressed in marrow MSCs per unit volume of the final hydrogel. The growth factor loading concentrations were 10 and $100 \mathrm{ng} / \mathrm{mL}$ for TGF- $\beta 1$ and IGF-1, respectively. The release kinetics of both growth factors from OPF hydrogel composites incorporating gelatin microparticles of the same composition as that examined in this study and prepared by crosslinking of the same type of OPF macromer as studied here, but with a
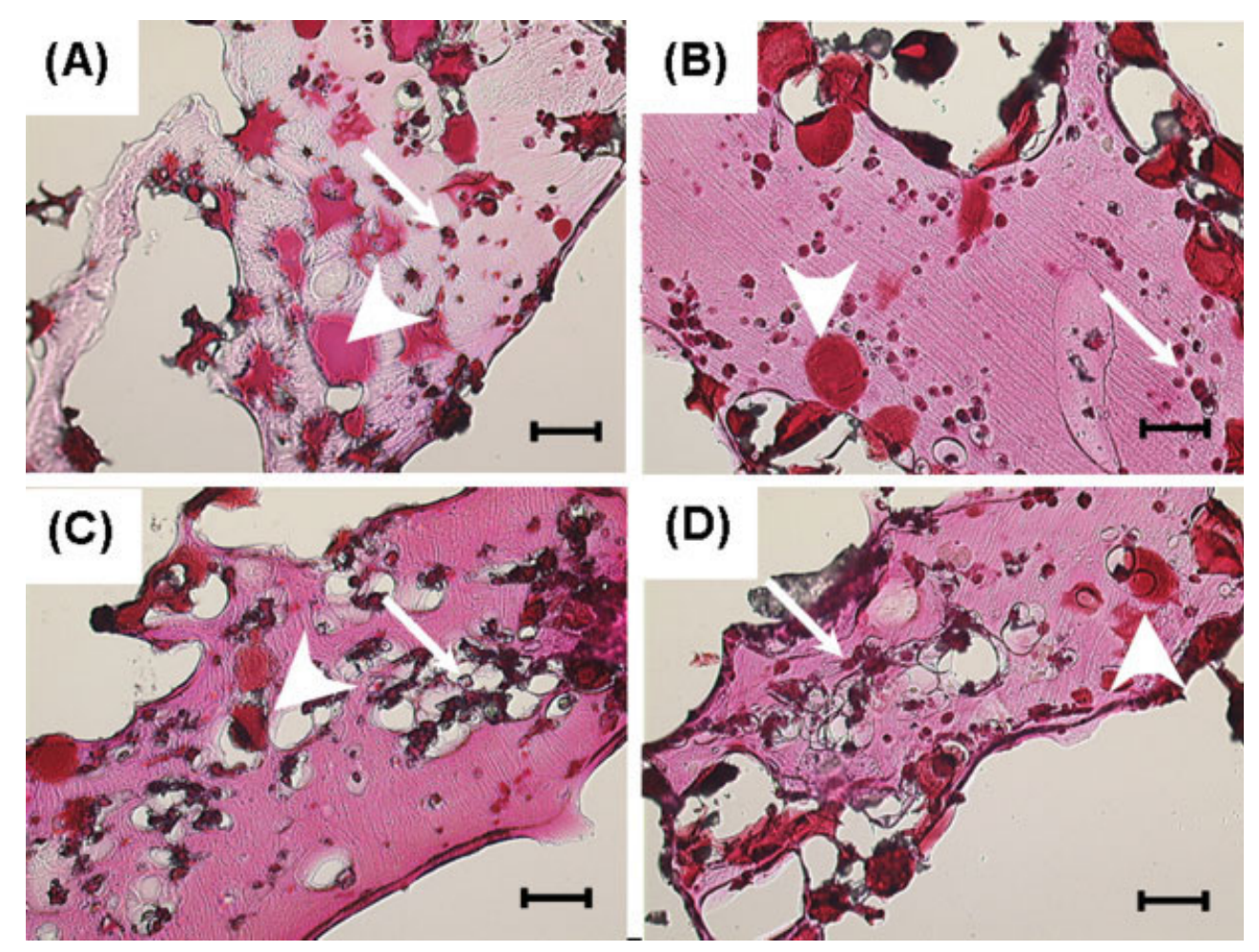

Figure 6. Cross-sections of OPF hydrogel composites encapsulating rabbit marrow MSCs/both IGF-1-loaded MPs and TGF- $\beta 1$-loaded MPs at day 0 (A), rabbit marrow MSCs/blank MPs at day 14 (B), rabbit marrow MSCs/ IGF-1-loaded MPs at day 14 (C), or rabbit marrow MSCs/both IGF-1-loaded MPs and TGF- $\beta 1$-loaded MPs at day 14 (D). Sections were stained with Safranin-O. Small arrows indicate rabbit marrow MSCs and large arrows indicate MPs in hydrogel composites. Scale bar represents $100 \mu \mathrm{m}$. [Color figure can be viewed in the online issue, which is available at www.interscience. wiley.com.] 
different crosslinking agent has been previously investigated, however, in the absence of any encapsulated cells. ${ }^{9,12}$ Although we recognize that the presence of encapsulated cells may affect the release kinetics of growth factors, the results of the previous studies can provide an estimate of the growth factor release profiles and local concentrations experienced by encapsulated cells.

A qualitative assessment of encapsulated cell morphology and aggregation was conducted at day 14 by confocal laser scanning microscopy after staining with calcein AM and ethidium homodimer-1. Calcein AM stains live cells with green fluorescence and ethidium homodimer-1 stains dead cells with red fluorescence. As seen in Figure 1, live rabbit marrow MSCs were distributed throughout OPF hydrogels with a sparse distribution of dead rabbit marrow MSCs. Interestingly, rabbit marrow MSCs coencapsulated with IGF-1-loaded MPs showed a tendency for cell aggregation [Fig. 1(B,C)], while rabbit marrow MSCs encapsulated without IGF-1-loaded MPs formed small clusters [Fig. 1(A)], suggesting that release of IGF-1 promoted the aggregation of rabbit marrow MSCs. The importance of strong cell-cell interaction and spherical morphology for chondrogenesis of MSCs has been previously reported. Strong cell-cell interactions mediated by $\mathrm{N}$-cadherin and integrins convert MSCs into prechondroblasts at the early mesenchymal condensation stage during limb development. ${ }^{20,21}$ This aggregation could be due to cell migration in OPF hydrogels rather than cell proliferation, since DNA assay results showed no increase in DNA content by day 14. Another explanation of the cell aggregation could be that the proliferation of MSCs was limited in a specific area in the hydrogels with concomitant death of cells in other regions, related to an increased concentration of IGF-1 in particular areas. This cell aggregation was more evident around gelatin microparticles. Figure 2 shows the $z$-axial projection stack image of rabbit marrow MSCs around gelatin microparticles. As shown in Figure 2(B), rabbit marrow MSCs appeared to attach to the surface of gelatin microparticles. However, rabbit marrow MSCs did not show the typical cell spreading shown by cells cultured in 2D plates. This result indicates that although gelatin microparticles provide sites for cell attachment, the OPF hydrogel surrounding them enables them to maintain their round morphology.

Results of the DNA assay showed a decrease in DNA content in all groups over the culture period, indicating that cell death may have occurred during the encapsulation process. ${ }^{9,22}$ A previous study with rabbit marrow MSCs showed a similar decrease of DNA content when encapsulated in OPF hydrogels. ${ }^{10}$ The highest decrease of DNA content was found in groups treated with TGF- $\beta 1$, suggesting that IGF-1 inhibited cell death in OPF hydrogel composites.

To examine the chondrogenic differentiation of rabbit marrow MSCs, the relative gene expressions of collagen type II, aggrecan, and collagen type I were analyzed. Collagen type II and aggrecan gene expression are markers of chondrogenic differentiation of MSCs, while collagen type I gene is mainly expressed by fibroblasts and other cell types. ${ }^{23}$ Gene expression was normalized to a housekeeping gene and expressed as a fold ratio as compared with the gene expression of control samples prepared at day 0 (rabbit marrow MSCs and blank MPs). At day 14, the highest increase in collagen type II expression was found in groups containing TGF- $\beta 1$-loaded MPs with or without IGF-1-loaded MPs [Fig. 4(A)]. However, the incorporation of IGF-1-loaded MPs did not promote collagen II gene expression of rabbit marrow MSCs as compared with groups containing TGF-B1-loaded MPs only. Aggrecan expression also showed a similar result as follows: a significant increase in aggrecan gene expression was found in groups with TGF- $\beta 1$-loaded MPs at day 14 regardless of the presence of IGF-1-loaded MPs [Fig. 4(B)]. These results suggest that TGF- $\beta 1$ mainly contributed to the upregulation of chondrocyte-specific genes for rabbit MSCs encapsulated in OPF hydrogel composites, even though IGF-1-loaded MPs promoted the cell aggregation.

The production of GAGs, which are associated with the chondrocytic phenotype of cultured cells, was also measured to examine the chondrogenic differentiation of rabbit marrow MSCs. As shown in Figure 5, the amount of GAG/DNA content was $\sim 5 \mu \mathrm{g} / \mu \mathrm{g}$ which is similar to that in other studies using rabbit MSCs for culture periods of 2 weeks ${ }^{24,25}$; however, no significant differences in GAG/DNA content were found between groups over the culture period. In accordance with these measurements, although histology images (Fig. 6) demonstrated rounded rabbit marrow MSCs during the culture period and strong cell aggregation in groups with IGF1-loaded MPs at day 14, there were no differences in GAG staining between groups at day 14. Contrary to our expectation, the incorporation of IGF-1-loaded MPs did not promote GAG production. This result may be due to the low concentration of IGF-1 loaded into the gelatin microparticles. In this experiment, IGF-1 was used only for loading MPs unlike other studies in which medium containing $100 \mathrm{ng} / \mathrm{mL}$ IGF-1 was changed every 2 or 3 days. ${ }^{19}$ Since TGF$\beta 1$ and IGF-1 promoted the upregulation of chondrocyte-specific genes and cell aggregation, respectively, one cannot assume a substantial decrease in the activity of loaded growth factors during the culture period. Alternatively, rabbit marrow MSCs of passage three might not be able to produce as much ECM as 
chondrocytes in vitro without further treatments even if they exhibited chondrocyte specific gene expression.

\section{CONCLUSION}

Rabbit marrow MSCs encapsulated in OPF hydrogels with IGF-1-loaded MPs, TGF- $\beta 1$-loaded MPs, or the combination of both remained viable and maintained a round morphology over a 14-day culture period. The presence of IGF-1-loaded MPs promoted the aggregation of rabbit marrow MSCs within the hydrogels. Additionally, when rabbit marrow MSCs were encapsulated with microparticles loaded with TGF- $\beta 1$, the cells exhibited an upregulation of chondrocyte-specific genes such as collagen type II and aggrecan. These results suggest that both IGF- 1 and TGF- $\beta 1$ loaded into gelatin microparticles were effectively delivered to rabbit MSCs encapsulated in hydrogel composites to promote the upregulation of chondrocyte-specific genes.

\section{References}

1. Temenoff JS, Mikos AG. Review: Tissue engineering for regeneration of articular cartilage. Biomaterials 2000;21:431440.

2. Kuo CK, Li WJ, Mauck RL, Tuan RS. Cartilage tissue engineering: Its potential and uses. Curr Opin Rheumatol 2006; 18:64-73.

3. Moutos FT, Freed LE, Guilak F. A biomimetic three-dimensional woven composite scaffold for functional tissue engineering of cartilage. Nat Mater 2007;6:162-167.

4. Hwang NS, Varghese S, Zhang Z, Elisseeff J. Chondrogenic differentiation of human embryonic stem cell-derived cells in arginine-glycine-aspartate-modified hydrogels. Tissue Eng 2006;12:2695-2706.

5. Kisiday J, Jin M, Kurz B, Hung H, Semino C, Zhang S, Grodzinsky AJ. Self-assembling peptide hydrogel fosters chondrocyte extracellular matrix production and cell division: Implications for cartilage tissue repair. Proc Natl Acad Sci USA 2002;99:9996-10001.

6. Lee KY, Mooney DJ. Hydrogels for tissue engineering. Chem Rev 2001;101:1869-1879.

7. Holland TA, Mikos AG. Advances in drug delivery for articular cartilage. J Control Release 2003;86:1-14.

8. Holland TA, Tessmar JK, Tabata Y, Mikos AG. Transforming growth factor- $\beta 1$ release from oligo(poly(ethylene glycol) fumarate) hydrogels in conditions that model the cartilage wound healing environment. J Control Release 2004;94:101114.

9. Park H, Temenoff JS, Holland TA, Tabata Y, Mikos AG. Delivery of TGF- $\beta 1$ and chondrocytes via injectable, biodegradable hydrogels for cartilage tissue engineering applications. Biomaterials 2005;26:7095-7103.

10. Park H, Temenoff JS, Tabata Y, Caplan AI, Mikos AG. Injectable biodegradable hydrogel composites for rabbit marrow mesenchymal stem cell and growth factor delivery for cartilage tissue engineering. Biomaterials 2007;28:3217-3227.

11. Elisseeff J, McIntosh W, Fu K, Blunk BT, Langer R. Controlled-release of IGF-I and TGF- $\beta 1$ in a photopolymerizing hydrogel for cartilage tissue engineering. J Orthop Res 2001; 19:1098-1104.

12. Holland TA, Tabata Y, Mikos AG. Dual growth factor delivery from degradable oligo(poly(ethylene glycol)fumarate) hydrogel scaffolds for cartilage tissue engineering. J Control Release 2005;101:111-125.

13. Holland TA, Bodde EW, Baggett LS, Tabata Y, Mikos AG, Jansen JA. Osteochondral repair in the rabbit model utilizing bilayered, degradable oligo(poly(ethylene glycol)fumarate) hydrogel scaffolds. J Biomed Mater Res Part A 2005;75:156167.

14. Jo S, Shin H, Shung AK, Fisher JP, Mikos AG. Synthesis and characterization of oligo(poly(ethylene glycol)fumarate) macromer. Macromolecules 2001;34:2839-2844.

15. Livak HJ, Schmittgen TD. Analysis of relative gene expression data using real-time quantitative PCR and the 2(-Delta Delta C(T)) method. Methods 2001;25:402-408.

16. Farndale RW, Buttle DJ, Barrett AJ. Improved quantitation and discrimination of sulphated glycosaminoglycans by use of dimethylmethylene blue. Biochim Biophys Acta 1986;883: 173-177.

17. Holland TA, Bodde EW, Cuijpers VM, Baggett LS, Tabata Y, Mikos AG, Jansen JA. Degradable hydrogel scaffolds for in vivo delivery of single and dual growth factors in cartilage repair. Osteoarthritis Cartilage 2007;15:187-197.

18. Fukumoto T, Sperling JW, Sanyal A, Fitzsimmons JS, Reinholz GG, Conover CA, O’Driscoll SW. Combined effects of insulin-like growth factor-1 and transforming growth factor- $\beta 1$ on periosteal mesenchymal cells during chondrogenesis in vitro. Osteoarthritis Cartilage 2003;11:55-64.

19. Longobardi L, O'Rear L, Aakula S, Johnstone B, Shimer K, Chytil A, Horton WA, Moses HL, Spagnoli A. Effect of IGF-I in the chondrogenesis of bone marrow mesenchymal stem cells in the presence or absence of TGF- $\beta$ signaling. J Bone Miner Res 2006;21:626-636.

20. Hall BK, Miyake T. Divide, accumulate, differentiate: Cell condensation in skeletal development revisited. Int J Dev Biol 1995;39:881-893.

21. Haas AR, Tuan RS. Chondrogenic differentiation of murine C3H10T1/2 multipotential mesenchymal cells. II. Stimulation by bone morphogenetic protein-2 requires modulation of N-cadherin expression and function. Differentiation 1999;64: 77-89.

22. Temenoff JS, Park H, Jabbari E, Sheffield TL, LeBaron RG, Ambrose CG, Mikos AG. In vitro osteogenic differentiation of marrow stromal cells encapsulated in biodegradable hydrogels. J Biomed Mater Res Part A 2004;70:235-244.

23. Sobajima S, Shimer AL, Chadderdon RC, Kompel JF, Kim JS, Gilbertson LG, Kang JD. Quantitative analysis of gene expression in a rabbit model of intervertebral disc degeneration by real-time polymerase chain reaction. Spine J 2005;5:14-23.

24. Park SH, Sim WY, Park SW, Yang SS, Choi BH, Park SR, Park K, Min BH. An electromagnetic compressive force by cell exciter stimulates chondrogenic differentiation of bone marrow-derived mesenchymal stem cells. Tissue Eng 2006;12: 3107-3117.

25. Toh WS, Liu H, Heng BC, Rufaihah AJ, Ye CP, Cao T. Combined effects of TGF- $\beta 1$ and BMP2 in serum-free chondrogenic differentiation of mesenchymal stem cells induced hyaline-like cartilage formation. Growth Factors 2005;23:313321. 